\title{
SISTEMATIZAÇÃO DA ASSISTÊNCIA DE ENFERMAGEM AO PACIENTE EM SÍNDROME DA ABSTINÊNCIA ALCOÓLICA NO PRONTO SOCORRO
}

[Systematization of nursing care to patients with alcoholic withdrawal syndrome in emergency units]

\author{
[Sitematización de la asistencia de enfermería al paciente con síndrome de la abstinencia \\ alcohólica en la unidad de emergencia]
}

José Raimundo Pereira dos Santos* Isaac R. M arques**

RESUMO: Este trabal ho trata de uma pesquisa bibliográfica cujo objetivo foi identificar o corpo de conhecimentos essenciais para prestar assistência de enfermagem a pacientes portadores da Síndrome da A bstinência A Icoólica na unidade de Pronto Socorro. A fonte principal de dados foi a B ase de Dados LILACS, usando-se expressões de pesquisa pertinentes ao tema. Para compor um corpo de conhecimentos essenciais na assistência, os resultados identificaram a necessidade de usar escalas de avaliação da síndrome, ter conhecimentos sobre as manifestações clínicas, saber as terapêuticas existentes e as respectivas intervenções. Concluiu-se que a identificação deste corpo de conhecimentos pode contribuir para dispensar-se uma assistência de enfermagem efetiva a pacientes portadores desta síndrome.

PALAVRAS-CHAVE: A Icoolismo; A taques por abstinência de álcool; Psiquiatria; Processos de enfermagem.

\section{INTRODUÇÃO}

0 ál cool é uma das poucas drogas psicotrópicas que têm seu consumo admitido pela sociedade, esse é um dos motivos pelo qual é encarado de forma diferenciada quando comparado com as demais drogas. A pesar de sua ampla aceitação social, o consumo de bebida alcoólica, quando excessivo, passa a ser um problema, pois além dos inúmeros acidentes de trânsito e da violência, pode, a longo prazo, provocar quadro de dependência conhecido como alcoolismo. D esta forma, o consumo inadequado do ál cool se constitui em um importante problema de saúde pública, que acarreta al tos custos para sociedade, envolvendo questões clínicas, psicológicas, profissionais e familiares ${ }^{(1)}$.

A demanda dos serviços de Pronto Socorro por par-

\footnotetext{
*A luno do 4ㅇ, ano da Faculdade de Enfermagem da U niversidade de Santo A maro.

**Professor A djunto da FA CEF-UNISA. O rientador do trabalho
}

te dos pacientes al coólatras corresponde de 12,5 a $36,7 \%$ das admissões ${ }^{(2)}$ e apesar da alta prevalência, o alcoolismo não é diagnosticado nas consultas e internações ${ }^{(3)}$. Geralmente, estes pacientes são atendidos por suas queixas físicas agudas. Esta demanda é ainda maior quando associada às admissões nos serviços de emergência por outras patologias e, caracteriza-se, no geral, por revelar pacientes com faixa etária de 43 anos ${ }^{(4)}$. Desta forma, há uma grande probabilidade desse tipo de paciente evoluir para Síndrome da A bstinência A Icoólica (SAA) dentro do próprio hospital, estando, assim, suscetível a uma série de riscos e complicações.

Os profissi onais de enfermagem, quando se trata do paciente em SAA, não têm o devido preparo para prestar assistência adequada ou eficiente; têm dificuldades para exercer sua função e realizar os cuidados necessários. I sso pode dever-se à sua formação ou denotar comodismo diante do problema ${ }^{(5)}$.

Diante de tal contexto, este trabal ho tem por objetivo agregar um corpo de conhecimentos essenciais, construídos a partir de revisão bibliográfica, cuja finalidade é revelar as proposições teóricas sobre o tema proposto, de modo a permitir ao profissional enfermeiro propiciar uma melhor assistência ao paciente em SAA.

\section{METODOLOGIA}

Trata-se de uma pesquisa bibliográfica baseada em consulta sistemática a bases de dados (bibliográficos) e no acesso não-sistemático a materiais em bibliotecas locais e outros disponíveis na web, localizados por meio de ferramentas de busca. Para a consulta sistemática, foi utilizada a B ase de Dados Bibliográficos da LILACS com uso da expressão de pesquisa abstinência alcoólica, assistência de enfermagem, enfermagem psiquiátrica ealcoolismo, sem o estabelecimento de limites de tipo de publicação, data da publicação ou idioma, o que se justifica por ser a problemática do al coolismo antiga na sociedade. $\mathrm{N}$ a busca não- 
sistemática foram consul tados al eatoriamente materiais de obras referenciais como livros-texto na área de Psiquiatria. Outros materiais foram acessados a partir de sua citação por autores dos trabalhos encontrados na pesquisa bibliográfica sistemática.

Os materiais recuperados foram classificados de acordo com o título e incluídos em subgrupos para compor o desenvol vimento do tema proposto. Para constituir o corpo de conhecimentos necessários para a assistência de enfermagem, considerando-se a necessidade de uniformização ou padronização de linguagem, foram utilizadas as classificações NA NDA ${ }^{{ }^{(6)}}$ (N orth-A merican N ursing Diagnoses A ssociation - A ssociação N orte-A mericana de Diagnósticos de Enfermagem) para diagnósticos de enfermagem e NIC (7) (Nursing Interventions Classification - Classificação de Intervenções de Enfermagem) para as intervenções de enfermagem.

\section{RESULTADOSE DISCUSSÃO}

\subsection{CARACTERIZA ÇÃ O DA AM OSTRA}

A pesquisa bibliográfica junto à Base da Dados da LILA CS resul tou em 67 trabal hos, dos quais 16 foram selecionados por atenderem aos critérios de inclusão do estudo. A discriminação do quantitativo apresenta-se na Tabela 1.

\subsection{GENERA LIDA DES SOBRE ALCOOLISMO}

O abuso e dependência de ál cool são chamados, ha- bitualmente, de alcoolismo ${ }^{(8)}$. Constitui um grave problema de saúde pública mundial, que acarreta sérios problemas físicos, mentais, sociais ${ }^{(9)}$. 0 álcool é a substância psicoativa mais utilizada em todo mundo, sendo o principal problema de saúde mental principalmente entre homens, com prevalência em torno de $15 \%$. Estima-se que no B rasil existam atualmente cerca de 18 milhões de al coolistas ${ }^{(10)}$, desta forma 0 uso de álcool deve ser investigado junto a todos os pacientes que procuram atendimento de saúde ${ }^{(3)}$.

\subsection{FISIOPATOLOGIA DA SAA}

A SAA resulta de um processo neuroadaptativo do sistema nervoso central, demonstrada por meio da hiperatividade noradrenérgica e glutamatérgica, da hipoatividade dopaminérgica e GA BA égica e do aumento da densidade de canais de cálcio tipo $L$, responsáveis todos pela sintomatologia ${ }^{(11)}$.

\subsection{MANIFESTA ÇÕES CLINICAS DA SAA}

A SA A é uma condição patológica caracterizada pela interrupção ou diminuição voluntária ou involuntária na ingesta habitual do álcool, revelada por um conjunto de sinais e sintomas como: tremores, ansi edade, irritabilidade, sudorese, aumento de pressão arterial, taquicardia, cefaléia, náuseas, vômitos; podendo ser precipitada por infecções, mal estado nutricional, desidratação, depressão, vulnerabilidade genética, padrão e tempo de consumo. Desenvolve-se de 7 a 8 horas após a interrupção ou dimi-

Tabela 1 - Caracterização e classificação dos materiais acessados, Santo A maro.

\begin{tabular}{lccccc}
\hline Classificação & \multicolumn{3}{c}{ Tipo de Publicação } & \multirow{2}{*}{ Total } \\
\cline { 2 - 4 } & Artigo & Tese & Livro & Outros* & \\
\hline Generalidades sobre alcoolismo & 1 & & 2 & 1 & 4 \\
Fisiopatologia da SAA & & & 1 & & 1 \\
Manifestações clínicas da SAA & 1 & 1 & & 2 \\
Diagnóstico do alcoolismo & 2 & & 1 & & 3 \\
Classificação da SAA & & & & 1 \\
Complicações da SAA & 1 & & 2 & 1 & 3 \\
Possíveis problemas clínicos & & & 2 & 1 & 3 \\
decorrentes do alcoolismo & & & & & 3 \\
Tratamento & 2 & & & 4 & 30 \\
Assistência de enfermagem & 7 & 2 & 1 & & 3 \\
\hline Totais & 14 & 2 & 10 & 4
\end{tabular}

*Outros: Livretos, M anuais e web sites. 
nuição da ingesta do álcool ${ }^{(8,12)}$. Quando se trata do tempo de duração da SA A há controvérsias entre as literaturas (2 a 7 dias ou 2 semanas), porém, o que se observou com 0 estudo é que os fatores (precipitadores da SA A) acima relacionados têm uma nítida relação com o início do quadro, tempo de duração, término, gravidade e possíveis complicações.

\subsection{DIAGNÓSTICO DO ALCOOLISMO}

Segundo o M anual do A lcoolista do M inistério da Saúde ${ }^{(3)}$, os profissionais de saúde devem estar atentos para a possibilidade de um caso de alcoolismo sempre que 0 paciente apresentar: hálito etílico, olhos e face avermelhados, emagrecimento e inapetência, náuseas e vômitos, nervosismo, insônia, mialgia ou miastenia, convulsões, escoriações e fraturas repetidas, histórico de acidente de trânsito e trabalho, desemprego prolongado, má adaptação familiar/social e histórico de espancamento.

Para diagnóstico do alcoolismo e monitorização da abstinência há também diferentes exames laboratoriais como: Gama-glutami-transpeptidase (GGT) eleva-se na ingestão de álcool e no etilismo crônico, na abstinência acompanha-se de redução (normal em homens 9-50 unidade/litro); Volume Corpuscular M édio Eritrocitário (V CM) encontra-se elevado no al coólatra e se normaliza após 3 a 4 meses de abstinência (normal em homens 80-100 fento/litro); A spartato A minotransferase (A ST) está aumentada em indivíduos alcoolistas e reduz-se à metade após 7 dias de abstinência (normal em homens 10-30 unidade/litro) ${ }^{(3,13)}$.

U ma outra forma utilizada para a detecção do alcoolismo e, conseqüentemente, para identificação de um possível risco do desenvolvimento da SAA é o questionário CA GE (Cut-down A nnoyed Gultty Eye-opener) ${ }^{(3,14)}$. Respostas afirmativas a pelo menos duas das quatro questões do questionário reforçam a hipótese de alcoolismo no paciente.

\section{Quadro 1 - Conteúdo do questionário CA GE}

1. Alguma vez o(a) Sr.(a) sentiu que deveria diminuir a quantidade de bebida ou mesmo parar de beber?

2. As pessoas o(a) aborrecem porque criticam o seu modo de beber?

3. O(a) Sr.(a) sente-se culpado(a) pela maneira como costuma beber?

4. O(a) Sr.(a) costuma beber pela manhã, para diminuir o "nervosismo" e/ou a ressaca?

\subsection{CLASSIFICA ÇÃO DA SAA}

A escala recomendada para avaliar o nível de SA A é a Clinical Withdrawal Assessment Revised -CIWA-A rAvaliação Clínica R evisada daA bstinência (anexo 1). Trata-se de uma escala que contém 10 itens, cujo escore final classifica a gravidade da SA A e fornece subsídios para 0 planejamento das intervenções imediatas ${ }^{(11)}$.

\subsection{COM PLICA ÇÕES DA SAA}

0 D elírio Tremens (DT) caracteriza-se como complicação por indivíduos em SA A que apresentam: confusão mental, desorientação tempo/espaço, flutuação do nível de consciência, distúrbios senso-percepção, delírios ou alucinações vividas; pode instalar-se de forma abrupta ou geral mente no decorrer de dois ou três dias após ter ocorrido a diminuição ou interrupção da ingesta da droga. Há também quadros de SA A que evoluem para A lucinose A Icoólica, caracterizada por indivíduos que apresentam alucinações auditivas vividas (vozes, sinos, rugidos e cânticos), contudo, diferencia-se do DT pela clareza do nível de consciência e o fato de que ocorre logo após a queda do nível sérico de ál cool. A Iguns pacientes em SA A, em geral usuários crônicos, podem apresentar convul sões entre 6 e 36 horas. A presença dessa complicação deve al ertar o profissional para uma possível ocorrência de severas manifestações clínicas ${ }^{(15)}$. Porém, há literaturas que associam as convul sões a episódios que precedem o DT, as mesmas literaturas, no entanto, relatam alguns pontos divergentes em relação ao assunto ${ }^{(16,8)}$

\subsection{POSSIIVEIS PROBLEM AS CLIINICOS DECORREN- TES DO ALCOOLISMO}

Os profissionais de saúde muito freqüentemente não reconhecem a associação entre problemas clínicos comuns e o uso excessivo de bebida alcoólica. É importante que eles reconheçam problemas como: hepatopatias, depressão, neuropatias, pancreatite, úlceras, gastrite, hemorragia digestiva, diarréia, desnutrição, desidratação, anemia, hipoglicemia, imunodepressão, distúrbios de coagulação, hipertensão, pneumonia aspirativa e tubercul ose ${ }^{(17)}$, possibilitando desta forma, um tratamento integral e o melhor prognóstico possível.

A Síndrome de Wernicke é um processo agudo decorrente da fal ta de tiamina (vitamina B 1) no indivíduo alcoólatra e geral mente desnutrido; o paciente caracteriza-se por apresentar ataxia (alterações motoras), confusão mental, distúrbios oculares (nistatismo horizontal, paralisia ocuIar, reação mais lenta à luz e anisocoria). Q uando não tratado rapidamente com tiamina, o paciente pode evoluir para quadro crônico denominado de Síndrome de Korsakoff, caracterizada pela incapacidade de reter informações, déficit de memória e aprendizado. A administração de glicose antes da tiamina pode precipitar ou piorar a Síndrome de Wernicke ${ }^{(2,3)}$. 


\subsection{TRATAM ENTO}

Em relação à terapia farmacológica, os benzodiazepínicos têm sido a droga de primeira escolha $(18,19)$, pois apresentam grande faixa de segurança, podendo ser usados na forma oral ou parenteral. A lém disso, têm ação anticonvulsivante, promovem profilaxia eficaz do DT e apresentam meia-vida longa ( 24 a 36 h.). No caso de alterações significativas da função hepática, podem ser utilizados L orazepam ou O xazepan, por apresentarem metabolismo oxidativo reduzido (20).

As perdas hídricas no paciente com DT podem ser da ordem de 5000 a $6000 \mathrm{ml}$ por dia ${ }^{(21)}$; desse modo a reposição hidroeletrol ítica se faz necessária no paciente em SA A, acompanhada de monitoramento dos níveis de sódio, potássio e magnésio, assim como do tratamento vitamínico com tiamina, complexo B eácido fólico. 0 tratamento deve ser complementado, também, nas possibilidades do paciente, por uma dieta hipercalórica e, se necessário, pela reposição de glicose (2).

0 paciente em SA A deve ser colocado em ambiente individual, calmo, sem ruídos ou luzes excessivas e devese manter com el e conversação, para que conserve juízo da realidade ${ }^{(2,19)}$. 0 repouso no leito é indispensável e, se necessário acompanhado de contenções mecânicas ${ }^{(10)}$.

\subsection{ASSISTÊNCIA DE ENFERMAGEM}

$\mathrm{Na}$ enfermagem, quando se trata do paciente al coólatra, há muitos preconceitos morais e recriminações. Esse paciente não é da preferência assistencial dos enfermeiros e suas atitudes enquanto líderes servem de base para toda equipe ${ }^{(22)}$. A sociedade não suporta os indivíduos que se vitimam por vontade própria; sendo assim, é difícil para o enfermeiro, dissociar-se do estigma social que caracteriza o alcoolismo, o que constitui, também, um obstáculo a falta de comunicação entre esses profissionais e al coólatras (23).

U m outro estudo ${ }^{(24)}$ que reforça a idéia dos alcoólatras como pacientes não-preferenciais dos profissionais de enfermagem aponta o fato destes considerarem aqueles irrecuperáveis. Porém, tais informações mostram que atitudes de enfermeiros refletem as influências de seu meio pessoal, profissional e, provavelmente, de sua formação acadêmica sobre o tema.

Para Rosa ${ }^{25)}$, a qualidade da assistência de enfermagem ao paciente al coól atra é insatisfatória; são necessários conhecimentos sobre 0 assunto. Para isso é importante a formação de grupo de estudos, a supervisão do estado emocional e das atitudes dos profissionais, cursos de atualização e pesquisas. Há estudos ${ }^{(26,27)}$ que reforçam ainda 0 despreparo destes profissionais, cujo argumento é o fato de não haver uma formação acadêmica ideal para sua prática.

0 Enfermeiro é o profissional mais próximo do paciente alcoólatra, presente em todos os momentos desde sua chegada até sua saída do hospital ${ }^{(28)}$. Isso Ihe permite caracterizar estes pacientes, analisar suas al terações e evolução, sejam elas físicas ou mentais ${ }^{(29)}$. Por isso, o processo de Enfermagem é um instrumento facilitador da assistência, entretanto, necessita ser usado e avaliado com base nas teorias de Enfermagem, para que resulte em uma meIhor assistência ${ }^{(30)}$.

A lgumas medidas gerais podem melhorar a assistência de enfermagem ao paciente em SA A, como: observáIo continuamente e examiná-lo repetidas vezes, tomar precauções para que ele não se fira, promover um ambiente calmo e seguro, chamá-lo pelo nome, explicar os procedimentos terapêuticos, fornecer dados da realidade, procurar tranqüilizá-lo verbalmente, evitar movimentações desnecessárias e, se possível, favorecer a presença de um membro da família para que ele mantenha contato com realidade ${ }^{(16)}$.

Buscando a padronização quanto à assistência de enfermagem, no anexo 2 está apresentado o quadro com os diagnósticos segundo a NANDA ${ }^{(6)}$ e intervenções de enfermagem sugeridas pela NIC ${ }^{(7)}$.

\section{CONSIDERAÇÕES FINAIS}

Diante dos aspectos abordados neste estudo, conclui-se que é importante para o enfermeiro conhecer a dimensão dos problemas relacionados ao alcoolismo, entretanto, é necessário entender e identificar a SA A, conhecer os instrumentos utilizados para sua aval iação, as possíveis complicações, as terapias existentes e, principal mente, buscando obter padronização da assistência de enfermagem bem como do plano de cuidados, que se conheçam os diagnóstico de enfermagem e suas devidas intervenções.

É preciso, no entanto, que o enfermeiro se dissocie do estigma de preconceitos e recriminações que o acompanham e passe a adquirir e valorizar seus conhecimentos, colocando-os em prática e disseminando-os para os demais membros da equipe.

Val e ressal tar que a formação deste corpo de conhecimentos possibilita ao enfermeiro atender ao paciente em SA A, para que ele receba tratamento integral, favorecendo assim, um melhor prognóstico. 0 desmame do álcool é 0 objetivo terapêutico no tratamento da SAA, porém envolve riscos e complicações que necessitam de intervenções, monitoramento e constantes avaliações. A importância de conhecer sobre esta situação clínica faz-se necessária para os enfermeiros, uma vez que certamente irão se deparar com indivíduos que procuram o pronto socorro por outro motivo e acabam desenvolvendo SA A durante período de internação ou até mesmo durante período de curta observação. A lém de tudo, é crescente a demanda de pacientes alcoólatras, principal mente nesta unidade.

ABSTRACT: This paper is a literature review that aimed to identify a body of essential nursing knowledge to assess patients with A Icoholic Withdrawal Syndromein Emergency Units. Primary data source was LILACS database using 
pertinent descriptors. To compose the body of essential knowledge for nursing care, results identified a need to use assessment scales, to have knowledge about clinical manifestations, to know existing therapeutics, and its related interventions. It was concluded that the identification of this body of knowledge can contribute for an effective nursing care to patients with that syndrome.

KEY WORDS: A Icoholism; A Icohol withdrawal seizures; Psychiatry; N ursing process.

RESUMEN: Este artículo es una investigación bibliográfica que tuvo como objetivo identificar un cuerpo esencial de conocimientos de enfermería para el cuidado de pacientes con la Síndrome de la A bstinencia A I cohólica en una unidad de emergencia. Los datos primarios fueran encontrados en la Base de Datos LILACS con el uso de descriptores pertinentes. Para la composición del cuerpo de conocimientos para la asistencia de enfermería, los resultados identificaron la necesidad del uso de escalas de evaluación, tener conocimiento acerca elas manifestaciones clínicas, conocer las terapéuticas existentes y sus respectivas intervenciones. Se concluyó que la identificación de ese cuerpo de conocimientos puede contribuir para una asistencia de enfermería efectiva a pacientes con ese síndrome.

PALABRAS CLAVE: A Icoolismo; Crisis por abstinência de alcohol; Psiquiatria; Processos de enfermería.

\section{REFERÊNCIAS}

1. Centro B rasileiro de Informações sobre Drogas Psicotrópicas. Livreto informativo sobre drogas psicotrópicas. São Paulo: OBID; s/d.

2. Bernik M A. U rgências relacionadas ao abuso de alcool. In: Fortes J RA, Cardo W N . A lcoolismo: diagnóstico e tratamento. São Paulo: Savier; 1991. p. 179-188.

3. M inistério da Saúde (BR). Secretaria Nacional de Programa Especiais de Saúde. Divisão N acional da Saúde M ental. N ormas e procedimentos na abordagem do alcoolismo. B rasília: M inistério da Saúde; 1990.

4. Fráguas J unior R, M iguel Filho EC. A lcoolismo no hospital geral. In: Fortes JRA, Cardo WN. A lcoolismo: diagnóstico e tratamento. São Paulo: Sarvier; 1991. p. 272-80.

5. Castledine G. Nurses should know how to tackle alcohol withdrawal. B J N urs 2003;12(11):644.

6. NA N DA - N orth-A merican N ursing Diagnoses A ssociation. Diagnósticos de enfermagem da NA NDA - Definições e classificação 19992000. Porto A legre: A rtmed; 1999.

7. M CCloskey JC, BulecheK GM. Nursing interventions classification (NIC). 3. ed. Saint L ouis: M osby; 2000.

8. K aplan HI, Sadock BJ , G rebb JA. Transtornos relacionados a substâncias. In: K aplan HI, Sadock BS, G rebb JA. Compêndio de psiquiatria: ciências do comportamento e psiquiatria clínica. 7. ed. Porto A legre:
A rtes M édicas; 1997. p. 369-95.

9. Caldas N M , Sadigursky D. A Icoolismo: U ma doença com implicações sociais. Rev Baiana Enferm 2002; 15(1/2): 49-54.

10. Fontana A M . Transtornos mentais e de comportamentos associados ao uso de substâncias psicoativas. In: Fontana A M. M anual de clínica em psiquiatria. São Paulo: A theneu; 2005. p. 255-66.

11. M arques A C, Ribeiro M . Á Icool: abuso e dependência. In: Laranjeira $R$, Oliveira RA, Nobre M R, B ernardo W M, Castro LA GP, Korniol $I G$, et al. U suários de substância psicoativas: abordagem, diagnóstico e tratamento. 2. ed. São Paulo: Conselho Regional de M edicina do Estado de São Paulo; 2003. p. 29-48.

12. L lorens M. Síndrome de abstinência alcohólica. A rch M ed Int 1999; 21(1):17-22.

13. Deguti M M, Gonçalves LL. Marcadores biológicos do alcoolismo. Rev Psiquiatr Clín 2000; 27 (1): 5-9.

14. Zuardi AW, Corrêa AJ, Cáceres Vera PEF, Lama J, Ferraz CA. Valor do questionário CA GE na detecção precoce dos pacientes com risco para o desenvolvimento de síndrome de abstinência alcoólica, num hospital geral. Rev A BP-A PAL 1987; 9 (4): 157-60.

15. Laranjeiras R. A buso e dependência do álcool. Diagn Tratamento 1997; 2 (3): 43-50.

16. Castillo JCR, M iguel Filho EC. Delirium-tremens. In: Fortes JRA, Cardo W N . A I coolismo: diagnóstico e tratamento. São Paulo: Sarvier; 1991. p. 189-98.

17. B arros SGS, Gal perem B. Problemas clínicos comuns do al coolista. In: Ramos SP et al. A Icoolismo hoje. Porto Alegre: A rtes M edicas; 1987.

18. Ribeiro M S, Texeira Y B. 0 tratamento farmacológico do alcoolismo. J Bras Psiquiatr 1999; 48(8): 345-53.

19. Varela M , Tapia L, L a R oche P. Tratamiento del síndrome de privación alcoholica. Bol Hosp San J uan de Dios 1985; 32 (1): 47-52.

20. Laranjeiras R. Avaliar e tratar a abstinência do álcool. Disponível em URL: http://www.abead.com.br. (20 fev. 2005).

21. M oreira Filho PF, Carvalho M L. Tratamento do delirium tremens nos serviços de emergência. A rq Bras M ed 1986; 60 (1): 57-8.

22. Vargas D. A titudes de enfermeiros de hospital geral frente ao paciente alcoolista. [dissertação]. Ribeirão Preto: Escola de Enfermagem de Ribeirão Preto, Universidade de São Paulo; 2001.

23. Diniz AS, Ruffino M C. Influência das crenças do enfermeiro na comunicação com alcoolista. Rev L atino-am Enferm 1996; 4 (n. especial): 17-23.

24. Navarrete PR, Luis M AV. A ctitud de la enfermera de um complejo hospitalario em relación al paciente al coholico. R ev L atino-am Enferm 2004; 12(n. especial): 420-6.

25. Rosa AJS. A ssistência de enfermagem à alcoolistas: opinião de 21 enfermeiros [dissertação]. Ribeirão Preto: Escola de Enfermagem de Ribeirão Preto, Universidade de São Paulo; 1991.

26. Canzian K CS, Castro R CBR. R egistros bibliográficos sobre a importância do papel da Enfermeira em assistência aos portadores de transtornos mentais em pronto-socorro geral. Base On-line dos trabalhos 
de conclusão de curso - Enfermagem - UNISA 2003; 4: 33-40.

27. Souza A S, M arcolan JF. A tuação da equipe de enfermagem na assistência a pacientes com transtornos psiquiátricos em pronto socorro geral. Base On-line dos Trabalhos de Conclusão de Curso - Enfermagem - UNISA 2001; 2: 6-13.

28. A ssunção A N . A I coolismo e Saúde M ental: uma reflexão de enfermagem. Cogitare Enferm 1996; 1 (1): 24-7.
29. Leopardi MT, A ssunção NA. Representações sociais de enfermeiros de emergência sobre o alcoolista. Cogitare Enferm 1996; 1(1): 1523.

30. Barros JFV, Camata M W, Santos EC, M acieira M S. M etodologia da assistência de enfermagem desenvolvida com pacientes do programa de atendimento ao alcoolista. J. B ras Psiquiatr 2000 jul; 49 (7): 247-54.

\section{ANEXO I}

\section{AVALIAÇÃO CLÍNICA DE RETIRADA REVISADA (CIWA-AR) (11)}

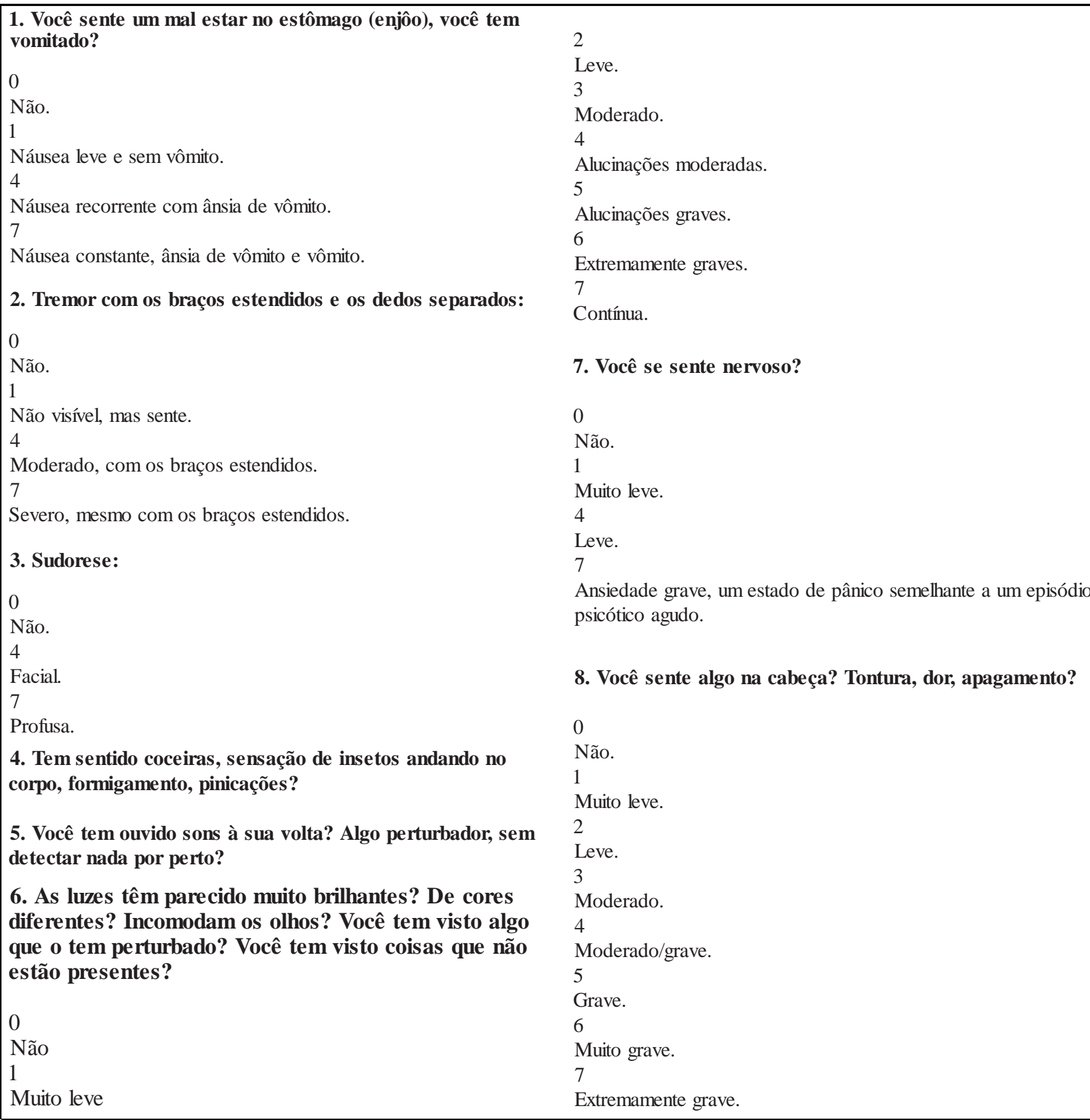


...continuação

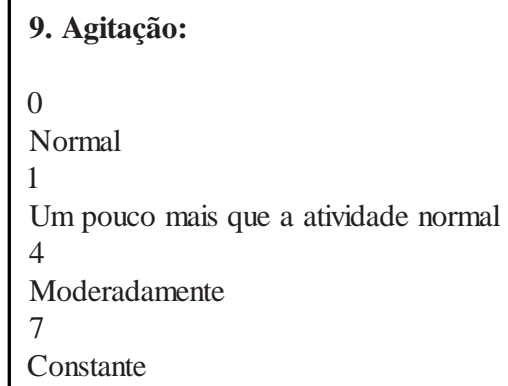

\section{Que dia é hoje? Onde você está? Quem sou eu? (Observação)}

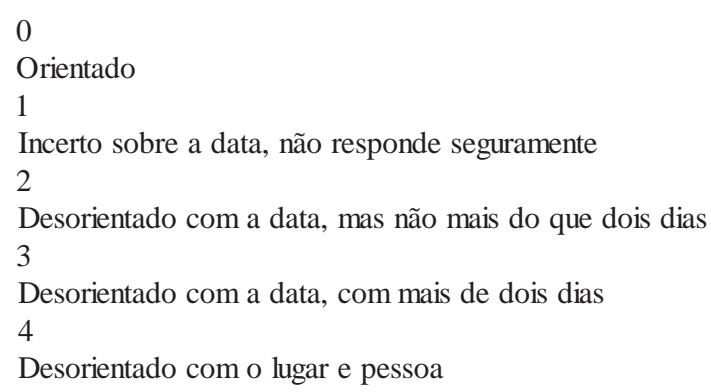

Escore Total:

\section{GRUPO DE DIAGNÓSTICOS DE ENFERM A GEM, SEGUNDO A NANDA ${ }^{(6)}$ E INTERVENÇÕES SUGERIDAS, SEGUNDO A NIC(7) PARA PACIENTES COM SAA}

\begin{tabular}{|c|c|}
\hline Diagnósticos & Intervenções \\
\hline $\begin{array}{l}\text { Nutrição alterada: ingestão menor do que as necessidades } \\
\text { corporais relacionada à anorexia }\end{array}$ & $\begin{array}{l}\text { - Controle/monitorização nutricional } \\
\text { - Controle/assistência no ganho de peso } \\
\text { - Controle de hipoglicemia } \\
\text { - Controle hídrico }\end{array}$ \\
\hline $\begin{array}{l}\text { Risco para déficit do volume de líquidos relacionado à perda } \\
\text { anormal de líquidos secundária ao vômito e à diarréia } \\
\text { Risco para lesão e/ou autolesão relacionado à desorientação, aos } \\
\text { tremores ou ao juízo prejudicado }\end{array}$ & $\begin{array}{l}\text { - Controle de infusão de líquidos } \\
\text { - Monitorização dos eletrólitos } \\
\text { - Manutenção de via intravenosa } \\
\text { - Controle do ambiente } \\
\text { - Prevenção de quedas } \\
\text { - Restrição física } \\
\text { - Monitorização neurológica }\end{array}$ \\
\hline $\begin{array}{l}\text { Risco para violência relacionado ao comportamento impulsivo, à } \\
\text { desorientação, aos tremores ou ao juízo prejudicado }\end{array}$ & $\begin{array}{l}\text { - Assistência no controle da raiva } \\
\text { - Redução da ansiedade } \\
\text { - Controle do ambiente: prevenção de violência } \\
\text { - Distração } \\
\text { - Intervenção nas crises } \\
\text { - Tratamento quanto ao uso da substância: álcool } \\
\text { - Restrição física } \\
\text { - Melhora na segurança } \\
\text { - Administração de medicação }\end{array}$ \\
\hline $\begin{array}{l}\text { Distúrbio do padrão do sono relacionado à irritabilidade, aos } \\
\text { tremores e aos pesadelos }\end{array}$ & $\begin{array}{l}\text { - Melhorar o sono } \\
\text { - Controle do ambiente } \\
\text { - Administração de medicamentos } \\
\text { - Redução da ansiedade } \\
\text { - Posicionamento apropriado no leito }\end{array}$ \\
\hline
\end{tabular}

Continua... 


\begin{tabular}{|c|c|}
\hline $\begin{array}{l}\text { Ansiedade relacionada à perda de controle, às perdas de } \\
\text { memória e ao medo da substância }\end{array}$ & $\begin{array}{l}\text { - Redução da ansiedade } \\
\text { - Controle comportamental } \\
\text { - Controle do ambiente }\end{array}$ \\
\hline $\begin{array}{l}\text { Estratégias ineficazes de resolução individual: raiva, dependência } \\
\text { ou negação relacionada à incapacidade de controlar } \\
\text { construtivamente os estressores sem álcool }\end{array}$ & $\begin{array}{l}\text { - Redução da ansiedade } \\
\text { - Apoio na tomada de decisões } \\
\text { - Apoio emocional } \\
\text { - Treinamento do controle dos impulsos } \\
\text { - Aconselhamento } \\
\text { - Intervenção nas crises }\end{array}$ \\
\hline $\begin{array}{l}\text { Interação social prejudicada relacionada à imaturidade emocional, } \\
\text { à irritabilidade, à ansiedade, ao comportamento impulsivo ou a } \\
\text { respostas agressivas }\end{array}$ & $\begin{array}{l}\text { - Melhorar a socialização } \\
\text { - Melhorar a auto-estima } \\
\text { - Tratamento do uso de substância: álcool } \\
\text { - Controle comportamental }\end{array}$ \\
\hline $\begin{array}{l}\text { Isolamento social relacionado à perda do trabalho ou ao } \\
\text { retraimento dos outros }\end{array}$ & $\begin{array}{l}\text { - Terapia da atividade } \\
\text { - Apoio emocional } \\
\text { - Controle do ambiente } \\
\text { - Melhorar a socialização } \\
\text { - Facilitação de visitas }\end{array}$ \\
\hline $\begin{array}{l}\text { Padrões de sexualidade alterados relacionados à impotência/perda } \\
\text { de libido secundárias à alteração do autocuidado e ao abuso do } \\
\text { álcool }\end{array}$ & $\begin{array}{l}\text { - Aconselhamento sexual } \\
\text { - Redução da ansiedade } \\
\text { - Melhora da imagem corporal }\end{array}$ \\
\hline $\begin{array}{l}\text { Estratégias ineficazes de resolução familiar relacionadas ao } \\
\text { rompimento conjugal e à inconsistência no estabelecimento de } \\
\text { limites }\end{array}$ & $\begin{array}{l}\text { - Tratamento quanto ao uso de substância - álcool } \\
\text { - Apoio emocional } \\
\text { - Controle comportamental }\end{array}$ \\
\hline $\begin{array}{l}\text { Risco para controle ineficaz do regime terapêutico relacionado } \\
\text { com o conhecimento insuficiente sobre a condição, os tratamentos } \\
\text { disponíveis, as situações de alto risco e os recursos da } \\
\text { comunidade }\end{array}$ & $\begin{array}{l}\text { - Modificação de comportamento } \\
\text { - Reestruturação cognitiva } \\
\text { - Melhora no enfrentamento } \\
\text { - Intervenção nas crises } \\
\text { - Apoio emocional } \\
\text { - Apoio à familia } \\
\text { - Aconselhamento nutricional } \\
\text { - Identificação de riscos } \\
\text { - Assistência na cessação do uso do álcool }\end{array}$ \\
\hline Risco para infecção & $\begin{array}{l}\text { - Proteção contra a infecção } \\
\text { - Controle de infecção }\end{array}$ \\
\hline Déficit no autocuidado para alimentação & $\begin{array}{l}\text { - Melhorar alimentação } \\
\text { - Controle nutricional } \\
\text { - Assistência no autocuidado: alimentação }\end{array}$ \\
\hline Déficit no autocuidado para banho/higiene & $\begin{array}{l}\text { - Ajudar no banho } \\
\text { - Assistência no autocuidado: banho/higiene } \\
\text { - Melhora na imagem corporal } \\
\text { - Melhora da auto-estima }\end{array}$ \\
\hline Confusão aguda & $\begin{array}{l}\text { - Controle do delírio } \\
\text { - Redução da ansiedade } \\
\text { - Restrição física } \\
\text { - Melhora do sono } \\
\text { - Orientação quanto à realidade } \\
\text { - Prevenção de queda }\end{array}$ \\
\hline
\end{tabular}

